\title{
Sexually dimorphic facial features vary according to level of autistic-like traits in the general population
}

Syed Zulqarnain Gilani ${ }^{1 \dagger}$, Diana Weiting Tan ${ }^{2,5^{*}}$, Suzanna N Russell-Smith², Murray T Maybery ${ }^{2}$, Ajmal Mian ${ }^{1}$, Peter R Eastwood ${ }^{3}$, Faisal Shafait ${ }^{1}$, Mithran Goonewardene ${ }^{4}$ and Andrew JO Whitehouse ${ }^{2,5}$

\begin{abstract}
Background: In a recent study, Bejerot et al. observed that several physical features (including faces) of individuals with an autism spectrum disorder (ASD) were more androgynous than those of their typically developed counterparts, suggesting that ASD may be understood as a 'gender defiant' disorder. These findings are difficult to reconcile with the hypermasculinisation account, which proposes that ASD may be an exaggerated form of cognitive and biological masculinity. The current study extended these data by first identifying six facial features that best distinguished males and females from the general population and then examining these features in typically developing groups selected for high and low levels of autistic-like traits.
\end{abstract}

Methods: In study 1, three-dimensional (3D) facial images were collected from 208 young adult males and females recruited from the general population. Twenty-three facial distances were measured from these images and a gender classification and scoring algorithm was employed to identify a set of six facial features that most effectively distinguished male from female faces. In study 2, measurements of these six features were compared for groups of young adults selected for high $(n=46)$ or low $(n=66)$ levels of autistic-like traits.

Results: For each sex, four of the six sexually dimorphic facial distances significantly differentiated participants with high levels of autistic-like traits from those with low trait levels. All four features were less masculinised for high-trait males compared to low-trait males. Three of four features were less feminised for high-trait females compared to low-trait females. One feature was, however, not consistent with the general pattern of findings and was more feminised among females who reported more autistic-like traits. Based on the four significantly different facial distances for each sex, discriminant function analysis correctly classified $89.7 \%$ of the males and $88.9 \%$ of the females into their respective high- and low-trait groups.

Conclusions: The current data provide support for Bejerot et al.'s androgyny account since males and females with high levels of autistic-like traits generally showed less sex-typical facial features than individuals with low levels of autistic-like traits.

Keywords: Autism, Autism spectrum disorder, Hypermasculinisation, Gender defiant disorder, Facial features, Masculinity, Femininity, Raine study

\footnotetext{
* Correspondence: diana.tan@research.uwa.edu.au

${ }^{\dagger}$ Equal contributors

${ }^{2}$ Neurocognitive Development Unit, School of Psychology, University of

Western Australia, 35 Stirling Highway, Crawley, 6009 Perth, WA, Australia

${ }^{5}$ Telethon Kids Institute, University of Western Australia, 100 Roberts Road,

Subiaco, 6008 Perth, WA, Australia

Full list of author information is available at the end of the article
} 


\section{Background}

A set of distinct facial features characterises a number of neurodevelopmental disorders such as Down syndrome (small and flat nose, small mouth, [1]) and Williams syndrome (flat nose, wide mouth and broad forehead, [2]). Autism spectrum disorder (ASD) is a neurodevelopmental disorder characterised by impairments in social and communication abilities and patterns of repetitive and stereotypical behaviours [3]. However, while ASD has not traditionally been thought to be characterised by distinctive facial features, this belief has been challenged in recent years.

In an early study of face morphology, Hammond et al. [4] reported that boys with ASD presented with greater facial asymmetry compared to controls. This finding led to the suggestion that facial information may be crucial in signalling aberrant brain development. Based on previous reports indicating that the brain and face of an individual arise from the same ectoderm layer in the embryo [5,6], Aldridge et al. [7] hypothesised that facial structures might reflect atypical neural development among individuals with ASD. Aldridge et al. [7] reported a distinct facial phenotype among 64 boys with ASD when compared to 41 typically developing boys. Some facial features that distinguished the two groups were distances between inner and outer corners of the eyes (increased in ASD), breadth of mouth (increased) and length of upper face (increased).

The largest study of the facial features of people with ASD was conducted by Ozgen et al. [8] as part of physical examinations of 112 children with ASD (93 boys and 19 girls) and 112 typically developing children matched on age and sex. There was a strikingly high prevalence of one or more major morphological anomalies (for example, open mouth appearance) among children with ASD (ASD: $43.8 \%$ vs. controls: $12.5 \%$ ), and a greater proportion of children with ASD with one or more minor anomalies (for example, forehead prominence and face asymmetry; ASD: $98.2 \%$ vs. controls: $58.9 \%)$. Most notably, facial structure was the most prominent area of morphological abnormalities, though there appeared to be no consistent pattern of differences (for example, both unusually smaller and unusually larger mouth sizes were significantly more frequent in children with ASD compared to controls). Additionally, Ozgen et al. [8] reported that morphological abnormalities were more common in males with ASD compared to females with ASD. Therefore, the study provided some indication of sex differences in the prevalence of morphological abnormalities though one has to be cautious given the limited size of the female samples.

ASD is more prevalent in males compared to females, with a male-to-female ratio of approximately 4:1 [9]. The hypermasculinisation hypothesis attempts to account for this large gender disparity by suggesting that ASD is an extreme variant of male behaviour and cognition [10-12]. Under one instantiation of the hypothesis, the hypermasculinisation associated with ASD is said to be caused by the exposure to increased levels of prenatal testosterone in utero. Several studies have indicated that a higher level of prenatal testosterone (measured in amniotic fluid) is associated with more autistic-like traits reported by parents [13], less eye contact [14], poorer emotion recognition [15] and better performance in male-favouring cognitive tasks such as mental rotation [16] and disembedding [17]. Furthermore, lower second-to-fourth digit ratio, which is indicative of exposure to higher levels of prenatal testosterone [18], has been observed in individuals with ASD compared to typically developing controls [19,20]. Additionally, some studies have also found increased levels of postnatal testosterone among individuals with ASD relative to controls. Schmidtova et al. [21] reported increased levels of salivary testosterone among prepubertal (4 to 10 years old) and pubertal (11 to 18 years old) boys with ASD relative to comparison groups. In adults, Ruta et al. [22] reported elevated levels of androgens in the blood serum of men and women with ASD compared to typical controls. Given that both prenatal [23,24] and postnatal $[23,25]$ testosterone levels are related to masculine facial features, one would expect individuals with ASD to present with more masculinised features compared to neurotypical individuals.

However, a recent study of the physical features of people with ASD provided data that challenge the hypermasculinisation hypothesis. Bejerot et al. [26] undertook several physical measurements (for example, waist-tohip ratio, second-to-fourth digit ratio, head circumference and ankle circumference) of 50 adults diagnosed with ASD and 53 age-matched typically developed individuals, and also collected subjective ratings of photographs of their faces, voices and bodies using a 'gender coherence' scale. Females with ASD were found to have a less feminine pattern of physical features than typically developed females in terms of waist-to-hip ratio (higher), head circumference (larger) and ankle circumference (larger), as well as receiving lower 'gender coherence' ratings of their faces. In contrast, males with ASD showed a less masculine pattern of results than typically developed males on digit ratios (higher) and also received lower 'gender coherence' ratings of their bodies and voices. Based on these findings, Bejerot et al. [26] proposed that rather than be identified with hypermasculinisation, characteristics of ASD may be better conceptualised as being androgynous.

Taken together, there is now evidence that individuals with ASD possess a set of facial features that are distinct from the features of typically developing individuals $[4,7,8]$. However, the relationship between facial structure 
and autistic traits has yet to be explored with reference to masculinity and femininity using objective facial measurements. Such an investigation would provide novel evidence pertinent to the hypermasculinisation and androgyny hypotheses.

There is now wide agreement that autistic-like traits form a continuum in the general population with ASD representing the extreme end of the distribution $[27,28]$. To capitalise on this, we recruited adults from the general population selected for high and low levels of autistic-like traits and focused on differences in sexually dimorphic facial features in the two groups. There were two phases to our investigations. The aim of the first study was to establish, using a gender classification and scoring algorithm [29], the facial features that best differentiate samples of males and females drawn unselectively from the general population. Since facial structure changes with age [30] and ethnicity [31], the first study was conducted to identify the most sexually dimorphic facial features using participants of very similar age and background to the participants to be recruited for the second study. Recruitment for the second study then comprised screening young adults using the Autismspectrum Quotient (AQ) questionnaire [32] to select groups of high and low AQ scorers for each sex. Critical interest then centred on whether the sexually dimorphic facial features identified in study 1 differentiated the groups with high versus low levels of autistic-like traits for each sex.

The hypermasculinisation and androgyny hypotheses can be used to generate competing predictions for the current study. Based on hypermasculinisation, it would be expected that individuals with high levels of autisticlike traits would display more masculinised facial features for males and less feminised features for females than their same-sex counterparts with low levels of autistic-like traits. Conversely, based on the androgyny account and findings reported by Bejerot et al. [26], it would be predicted that men with high levels of autisticlike traits would display less masculine facial features than men with low levels of those traits, while women with high levels of autistic-like traits would display less feminine facial features than women with low levels of those traits.

\section{Study 1}

The aim of this study was to identify a set of facial features that best distinguish young adult males and females, using a gender classification and scoring algorithm [29]. We used three-dimensional (3D) images for all facial measurements and analyses because these are less susceptible to effects of illumination and pose variations and are capable of capturing facial surface information unlike twodimensional (2D) images [33]. Using 3D images and objective measurements of facial features, Burton et al. [34] found that nose protuberance was the best facial feature for differentiating male from female faces in samples aged 18 to 30 years. Other features include length of cheek and philtrum length. In a more recent study, Velemínská et al. [35] found that men $\left(M_{\text {age }}=21.1\right.$ years $)$ had smaller distances between the eyes, more deeply-set eyes with respect to facial plane, larger noses and wider chins compared to women $\left(M_{\text {age }}=21.6\right.$ years $)$. Similarly, among male and female adolescents aged 12 to 18 years old, Chakravarty et al. [36] found that male adolescents had larger facial distances in features such as forehead, chin jaw, nose length, nose protuberance and philtrum length when compared to female adolescents.

\section{Methods}

\section{Participants}

The 208 participants (107 males; 101 females; $M_{\text {age }}=$ 22.81 years, $\mathrm{SD}_{\text {age }}=0.63$ years) were recruited from the Western Australian Pregnancy Cohort (Raine) Study [37], an ongoing population-based longitudinal study. To control for the effects of ethnicity on face structure [31], only Caucasians were recruited for the study. Ethics approval was obtained from the Human Research Ethics Committee at the University of Western Australia, and written informed consent was obtained from each participant.

\section{Apparatus}

$3 \mathrm{D}$ images of the face were acquired using a 3dMDface system (3dMD, Atlanta, GA, USA) operated from a desktop computer in a room where lighting was kept constant. The $3 \mathrm{dMDface}$ system is a non-invasive imaging technology that produces $3 \mathrm{D}$ images $\left(180^{\circ}\right.$ ear-toear frontal view) using random light projection on the face of the participant, as well as combining multiple 2D images captured using colour and infrared cameras from two stereo camera viewpoints. The use of infrared cameras allows the distance between the system and the participant to be standardised for all images obtained. Previous studies have shown that facial analyses using images captured by a 3dMDface system are highly precise and replicable [38,39]. A more detailed description of the 3dMDface system is available from http://3dMD. com/3dMDface/.

\section{Procedure}

Participants sat in front of the 3dMDface scanner with the distance between the chair and scanner adjusted so their faces appeared in the middle of the computer screen. During the imaging process, participants fixated their gaze on a sticker pasted on the wall behind the scanner, maintained a neutral facial expression and kept their mouths closed. No accessories were worn for the 
imaging process and loose hair was pinned back from the face.

\section{Gender classification and scoring algorithm}

The algorithm is capable of identifying sexually dimorphic facial features (classification accuracy of 94\%) and assigning gender scores to 3D face images using objective measurement. The algorithm is briefly summarised in this paper and further details can be found in [29].

In the first stage of the analysis, 21 facial landmarks, defined by Farkas [40], were annotated on each image (see Figure 1) and 23 linear distances (see Table 1) were measured using Matlab. Next, the minimal redundancy maximal relevance algorithm was used to select features that were most relevant for distinguishing male and female faces. The final stage involved training a linear discriminant analysis (LDA) classifier [41] using the selected features. The training was performed using a tenfold validation technique in which nine folds were used for training the classifier and one fold for testing. The LDA classifier identified an optimal subset of facial features that most effectively and accurately classifies the $3 \mathrm{D}$ face images into their respective gender.

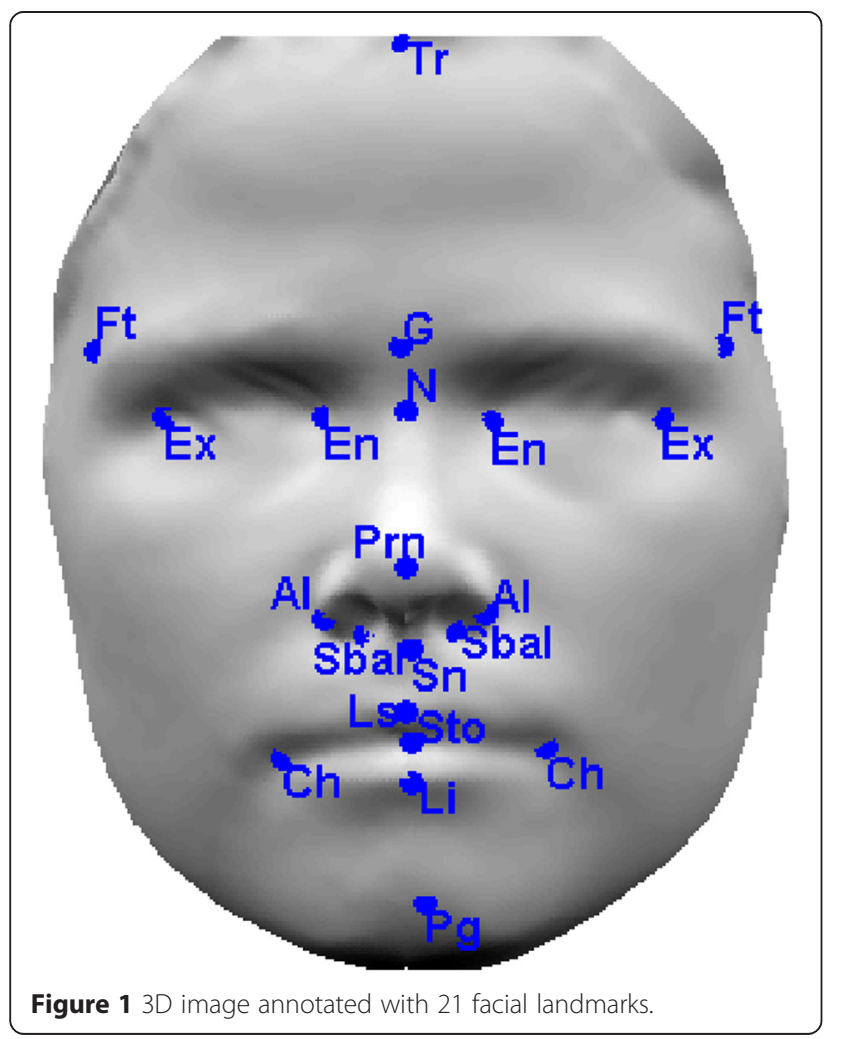

Table 1 Summary of facial landmarks and distances measured in study 1

\begin{tabular}{lll}
\hline Number & Landmark & Facial distance \\
\hline 1 & Ft-Ft & Forehead width \\
2 & Ex-Ex & Outer canthal width \\
3 & N-Prn & Nasal bridge length \\
4 & Sn-Prn & Nasal tip protrusion \\
5 & Sn-Ls & Philtrum length \\
6 & Al-Al & Nose width \\
7 & Ex-En (left) & Eye fissure length (left) \\
8 & Ex-En (right) & Eye fissure length (right) \\
9 & En-En & Intercanthal width \\
10 & Ch-Ch & Mouth width \\
11 & N-Sto & Upper facial height \\
12 & N-Sn & Nose height \\
13 & En-N (left) & Nasal root height (left) \\
14 & En-N (right) & Nasal root height (right) \\
15 & Sn-Sto & Upper lip height \\
16 & Ls-Sto & Upper vermillion height \\
17 & Sto-Li & Lower vermillion height \\
18 & Tr-G & Forehead height \\
19 & Sto-Pg & Mandible height \\
20 & Sbal-Sbal & Alar base width \\
21 & Sbal Prn & Alar length \\
23 & Lower profile height \\
\hline & &
\end{tabular}

\section{Statistical analysis}

Independent-samples $t$-tests were conducted to confirm that the linear distance for each of the selected facial features differed significantly between males and females.

\section{Results}

From the 23 linear distances measured, LDA identified a subset of six distances that provided the highest sex classification accuracy $(97.19 \%$ for males and $95.04 \%$ for females). These features were forehead width (Ft-Ft), outer canthal width $(E x-E x)$, nasal bridge length $(N-P r n)$, nasal tip protrusion $(S n-P r n)$, philtrum length $(S n-L s)$ and nose width $(A l-A l)$.

Independent-samples $t$-tests revealed that all six of these sexually dimorphic facial features were significantly larger in distance in males than in females (see Table 2): forehead width, $t(206)=14.30, P<.001, r^{2}=.50$; outer canthal width, $t(206)=3.75, P<.001, r^{2}=.06$; nasal bridge length, $t(206)=10.18, P<.001, r^{2}=.33$; nasal tip protrusion, $t(206)=9.24, P<.001, r^{2}=.29$; philtrum length, $t(206)=3.02, P=.003, r^{2}=.04$; nose width, $t$ $(206)=10.59, P<.001, r^{2}=.35$. 
Table 2 Means (and standard deviations) in millimetres of the critical facial distances for each sex

\begin{tabular}{llll}
\hline & Male $(\boldsymbol{n}=\mathbf{1 0 7})$ & Female $(\boldsymbol{n}=\mathbf{1 0 1})$ & $\boldsymbol{P}$ \\
\hline Ft-Ft: forehead width & $124.12(5.27)$ & $113.41(5.53)$ & $<.001$ \\
Ex-Ex: outer canthal width & $96.57(4.54)$ & $94.29(4.20)$ & $<.001$ \\
N-Prn: nasal bridge length & $47.34(3.77)$ & $42.12(3.62)$ & $<.001$ \\
Sn-Prn: nasal tip protrusion & $19.67(1.95)$ & $17.18(1.94)$ & $<.001$ \\
Sn-LS: philtrum length & $8.17(2.44)$ & $7.25(1.94)$ & .003 \\
Al-Al: nose width & $32.71(2.58)$ & $29.08(2.35)$ & $<.001$ \\
\hline
\end{tabular}

\section{Study 2}

Study 2 aimed to examine whether the six sexually dimorphic facial features identified in study 1 could distinguish, for each sex, faces of groups of individuals selected for high and low levels of autistic-like traits.

\section{Methods}

Participants

Based on the lower and upper quartiles of the score distribution of the Autism-spectrum Quotient (AQ; [32]), the cut-off scores for low and high scores were $\leq 10$ and $\geq 22$, respectively. These cut-off scores are comparable to those employed in previous studies reporting differences between high and low AQ groups (see, for example, [42]), and are extreme with reference to the nonclinical samples reviewed by Ruzich et al.[43], which yielded a weighted mean AQ of $16.94 \%$ and a $95 \%$ confidence interval of 11.6 to 20.0 for the 78 samples. Sixty-one (38 males) of the Raine participants (described in study 1 ) met the criterion for either high- or low-AQ group inclusion. These volunteers had completed the AQ in an earlier study [44] (mean age at time of testing $=19.74$ years, $\mathrm{SD}=0.79$ years $)$. An additional 51 Caucasian undergraduates (20 males; 31 females; $M_{\text {age }}=19.0$ years, $\mathrm{SD}=1.94$ years) who also met inclusion criteria were recruited from the University of Western Australia to increase sample sizes. The final sample comprised 112 young adults (58 males), and descriptive statistics are provided in Table 3.

\section{Autism-spectrum Quotient (AQ)}

The AQ [32] is a self-report questionnaire that assesses levels of autistic-like traits in the general population. Participants are provided with 50 statements (for example, 'People often tell me that I keep going on and on about the same thing.' and 'I am fascinated by numbers.')

Table 3 Descriptive statistics and critical facial distances for the pairs of high- and low-AQ groups for each sex

\begin{tabular}{|c|c|c|c|c|c|c|}
\hline & Males & & & Females & & \\
\hline & $\begin{array}{l}\text { Low-AQ } \\
(n=33)\end{array}$ & $\begin{array}{l}\text { High-AQ } \\
(n=25)\end{array}$ & $P$ & $\begin{array}{l}\text { Low-AQ } \\
(n=33)\end{array}$ & $\begin{array}{l}\text { High-AQ } \\
(n=21)\end{array}$ & $P$ \\
\hline AQ score & & & & & & \\
\hline $\mathrm{M}(\mathrm{SD})$ & $8.82(1.04)$ & $25.20(2.26)$ & & $6.58(1.60)$ & $26.48(3.63)$ & \\
\hline Range & 7 to 10 & 22 to 30 & & 1 to 8 & 22 to 35 & \\
\hline Age & & & & & & \\
\hline $\mathrm{M}(\mathrm{SD})$ & $19.24(1.20)$ & $19.72(2.01)$ & & $18.91(1.63)$ & $19.29(1.15)$ & \\
\hline Range & 17 to 22 & 17 to 26 & & 17 to 23 & 17 to 22 & \\
\hline Facial dista & & & & & & \\
\hline Forehea & & & & & & \\
\hline$M(S D)$ & $126.01(5.74)$ & $121.11(5.69)$ & .002 & $110.63(5.11)$ & $116.53(4.73)$ & $<.001$ \\
\hline Outer ca & & & & & & \\
\hline$M(S D)$ & $98.38(4.38)$ & $94.26(4.47)$ & .001 & $90.95(3.61)$ & $96.59(4.44)$ & $<.001$ \\
\hline Nasal br & & & & & & \\
\hline $\mathrm{M}(\mathrm{SD})$ & $49.37(4.38)$ & $46.17(3.69)$ & .005 & $43.83(3.00)$ & $40.40(3.64)$ & $<.001$ \\
\hline Nasal tip & & & & & & \\
\hline $\mathrm{M}(\mathrm{SD})$ & $21.62(1.94)$ & $18.65(2.29)$ & $<.001$ & $19.52(2.10)$ & $19.90(2.31)$ & .538 \\
\hline Philtrum & & & & & & \\
\hline $\mathrm{M}(\mathrm{SD})$ & $22.99(2.17)$ & $23.67(3.05)$ & .323 & $21.25(2.49)$ & $21.62(2.58)$ & .601 \\
\hline Nose wi & & & & & & \\
\hline $\mathrm{M}(\mathrm{SD})$ & $32.81(3.10)$ & $32.46(2.42)$ & .642 & $28.58(2.10)$ & $30.38(2.01)$ & .003 \\
\hline Facial area & & & & & & \\
\hline$M(S D)$ & $24,600(1,670)$ & $24,500(1,870)$ & .807 & $22,800(1,620)$ & $23,100(1,650)$ & .569 \\
\hline
\end{tabular}

$\mathrm{M}$, mean; SD, standard deviation; $A Q$, Autism-spectrum Quotient. 
and indicate how well each statement applies to them (score range: 0 to 50 ). The AQ has good test-retest reliability $(r=.70,[32])$ and its major factors (social skills, attention to patterns/details and communication: see [45]) correspond to key dimensions of ASD symptoms. The instrument reliably distinguishes ASD and neurotypical groups $[32,46]$ and high-AQ samples differ from their low-AQ counterparts in ways that mirror ASD versus control differences (for example, $[42,47,48]$ ).

\section{Apparatus and procedure}

The 3dMDface system and imaging procedure described in study 1 were also used in this study to obtain the 3D face images.

\section{Statistical analysis}

We annotated 12 facial landmarks on each image and measured the six distances that were found to be sexually dimorphic in study 1 . Independent-samples $t$-tests were conducted to compare the six distances between participants in the low- and high-AQ groups within each sex. Discriminant function analyses were then used to classify participants into their respective AQ groups based on the facial distances that were significantly different.

\section{Results}

Descriptive statistics for the face measurements are shown in Table 3. Four of the six distances were found to be significantly different between high- and low-AQ groups within each sex. Generally, males in the high-AQ group were found to have less masculine features (smaller distances) than males in the low-AQ group: forehead width, $t(56)=3.24, P=.002, r^{2}=.16$; outer canthal width, $t(56)=3.51, P=.001, r^{2}=.18$; nasal bridge length, $t(56)=2.95, P=.005, r^{2}=.13$; and nasal tip protrusion, $t(56)=5.34, P<.001, r^{2}=.34$. Females in the high-AQ group were found to have less feminine features (larger distances) than their low-AQ counterparts for three of the six features: forehead width, $t$ $(52)=4.26, P<.001, r^{2}=.26$; outer canthal width, $t$ $(52)=5.11, P<.001, r^{2}=.33$; and nose width, $t(52)=$ $3.12, P=.003, r^{2}=.16$. The only significant distance to not fit this pattern was nasal bridge length, with this feature shorter in length (more feminine) in the high-AQ females compared to the low-AQ females, $t$ $(52)=3.76, P<.001, r^{2}=.21$.

Since it is possible that body mass index could affect facial distances, a secondary analysis investigated whether there were differences in total facial area between the high- and low-AQ groups. Area of the face was calculated using the $3 \mathrm{D}$ point cloud that defined each face and the triangular connectivity between these points. Independent samples $t$-tests identified no group differences in total facial area for males $(P=.807)$ or females $(P=.569$; see Table 3$)$. This indicates that differences in facial distances were not due to differences in overall face size.

For each sex, the four significantly different distances were entered into a discriminant function analysis aimed at classifying participants into their high- and low-AQ groups. In males, all four facial distances were significant predictors of group membership, $X^{2}=40.7, P<.001$ (see Table 4 for standardised coefficients). Classification results showed that $89.7 \%$ (cross-validated percentage of $84.5 \%$ ) of the male participants were correctly classified into their respective AQ groups. In females, all four facial distances were also significant predictors, $X^{2}=29.0$, $P<.001$ (see Table 4) with $88.9 \%$ (cross-validated percentage of 79.6\%) of the women correctly classified into their AQ groups.

\section{Discussion}

The current study used 3D technology and a gender classification and scoring algorithm [29] to investigate the facial phenotypes of males and females in the general population, as well as young adults selected for high and low levels of autistic-like traits. Study 1 aimed to identify facial features that distinguished the faces of males and females in the general population. LDA identified a set of six facial distances capable of classifying the young adult male and female faces with an accuracy of $97.19 \%$ and $95.04 \%$, respectively. Several of the sexually dimorphic facial features found in the current study are consistent with previous findings, such as nasal tip protrusion [34], philtrum length [34,36] and the widths of forehead and nose $[35,36]$.

Study 2 then examined these six sexually dimorphic facial features in groups of males and females selected for high and low levels of autistic-like traits. Among males, four of the six facial features (forehead width, outer canthal width, nasal bridge length and nasal tip protrusion) were significantly less masculinised in the high-AQ group compared to the low-AQ group. Among females, four features were significantly different between

Table 4 Facial distances entered into discriminant function analysis for each sex

\begin{tabular}{lll}
\hline & \multicolumn{2}{l}{ Standardised coefficient } \\
\cline { 2 - 3 } Facial distances & Male & Female \\
\hline Forehead width & .41 & .67 \\
Outer canthal width & .44 & .80 \\
Nasal bridge length & .37 & -.59 \\
Nasal tip protrusion & .67 & Not entered \\
Nose width & Not entered & .49 \\
\hline
\end{tabular}

Standardised coefficients with an absolute value greater than .30 are considered significant predictors [69]. 
the high- and low-AQ groups. Three features (forehead width, outer canthal width and nose width) were less feminised in the high-AQ group compared to the low-AQ group while one feature (nasal bridge length) was more feminised in the high-AQ group. These findings indicate that individuals with high levels of autistic-like traits generally show less sex-typical facial characteristics than those with low levels of these traits. Intriguingly, however, the outcome for nasal bridge length among the females was in the opposite direction to the general pattern of results. Bruce et al. [49] argued that when observers classify faces as male or female, some of the features contributing to reliable classification, such as forehead width and nose protuberance, are processed locally (that is, independent of other features) while other features, such as nasal bridge length, are processed configurally (that is, relative to other features such as the width of the face). Although the current study examined objective measurements of faces rather than perceptual classification of sex, a possible extension of the current research would be to evaluate whether configurations of features that differentiate males and females assist in differentiating groups selected to differ in levels of autistic-like traits. The utility of ratios such as the ratio of nasal bridge length to width of the face would be of particular interest.

Results of the current study are generally consistent with Bejerot et al.'s [26] androgyny account and run counter to the hypermasculinisation hypothesis. In a recent study, Lai et al. [50] presented neuroanatomical data obtained from males and females with and without ASD. The neuroanatomy of ASD was found to be sex-dependent. More specifically, females with ASD showed a more 'masculinised' neuroanatomy compared to females without ASD. However, the brain masculinisation we would expect based on the hypermasculinisation account was not observed in males. Lai et al. made further investigations by comparing two sexually dimorphic brain structures that characterised 'feminisation' in males and found that one of the two brain areas showed more feminisation in males with ASD compared to those without ASD.

Brain masculinisation in females with ASD and feminisation in males with ASD reported by Lai et al. [50] is in line with the pattern of results reported in Bejerot et al. [26] and in the current study. Given that the development of the brain and face occurs in concert in utero [4], these results further strengthen the position that facial information may provide a crucial marker of aberrant neurodevelopment in ASD. In addition, these results also indicate that characteristics of ASD may manifest differently in males and females. While the hypermasculinisation hypothesis may account for masculinisation in females, the androgyny hypothesis may provide a more complete description of the characteristics of ASD in both sexes.

Nonetheless, some research outcomes have been consistent with the hypermasculinisation account and others not. Scott et al. [51] reported evidence of an association between higher AQ scores and more masculinised faces for males but not for females. While these results are difficult to reconcile with those observed in the current study, there are important methodological differences. For example, Scott et al. [51] obtained masculinity indices via subjective ratings, whereas the current study used objective markers of facial morphology. Furthermore, the face stimuli used by Scott et al. [51] were those of mid-AQ individuals that had been synthetically morphed towards composite facial averages derived from highand low-AQ participant samples. By contrast, the current study examined objective markers in the actual faces of people with high- or low-AQ, thus providing a more direct test of the hypotheses.

More broadly, while there has been reports of prenatal testosterone concentrations being positively associated with the development of autistic-like traits [13] and ASD [52] later in life, there is no evidence of a link between perinatal testosterone (measured from cord blood) and autistic-like traits [44]. Regarding postnatal testosterone, some studies reported a positive association between testosterone levels and autistic-like traits [22,53], while one study found no such association [54]. In Bejerot et al. [26], females with ASD were found to have higher levels of testosterone compared to female controls, but no difference was found in males.

In addition, there have been numerous studies that have reported hypermasculinised cognitive abilities for both males and females with ASD. For instance, individuals with ASD tend to perform better on malefavouring tasks (for example, mental rotation [16]) and perform worse on female-favouring tasks (for example, emotion recognition [15]). Although these findings based on cognitive data are difficult to reconcile with the current findings based on physical characteristics, an early study by Petersen [55] reported data that provides a link between masculinised cognition and androgynous physical features. Using masculinity-femininity ratings of images of adolescent bodies, Petersen found that males with bodies rated as less masculine (more androgynous) performed better on a spatial task (a male-favouring ability) but worse on a verbal task (a female-favouring ability) relative to males with more masculine body ratings. As for female adolescents, those with less feminine bodies showed better performance on the spatial task but equivalent performance on the verbal task, relative to those with more feminine bodies. Thus, for both sexes, androgynous body ratings were associated with a 
pattern of cognitive performance typically associated with masculinisation.

At present, the specific biological mechanisms that could underlie the development of androgynous facial features and their relationships with autistic traits remain unclear. Embryonic craniofacial development is genetically predetermined and can also be influenced by extrinsic environmental factors [56]. Potential candidate genes have been identified among some individuals with ASD [57]. It is possible that these genes not only express themselves in the form of autistic traits but also influence facial phenotypes of those with ASD. In addition, it is possible that fetal environment factors such as levels of prenatal testosterone influence both neurodevelopment and face development in concert.

To our knowledge, this is the first study to explore the degree of facial masculinity and femininity using objective measurement in healthy individuals selected for high and low levels of autistic-like traits. The strength of the study is that participants in both phases of the study were matched on age and background to ensure validity in the facial features assessed. The current study also employed the use of advanced face scanning technology and software algorithms to obtain reliable objective measurements and analyses of facial features.

However, three features of the study constrain interpretation of the results. First, while the AQ is a valid and efficient instrument to use in screening large numbers of neurotypical participants to recruit groups with high and low levels of autistic-like traits, it does not assess clinically significant symptoms. Future research of this kind would benefit from checking for ASD symptoms using an instrument such as the Autism Diagnostic Observation Schedule, Second Edition [58] or Autism Diagnostic Interview-Revised [59]. Second, the current study did not investigate clinical samples of individuals diagnosed with ASD. However, population-based studies have provided support for a smooth etiological continuum of autistic-like traits across the general population, with clinical ASD representing the extreme end of a quantitative distribution [60]. The current findings warrant future research to examine if similar results can be replicated in comparing ASD and neurotypical samples. Lastly, with the current study investigated facial features for Caucasian participants, our findings cannot be generalised to other ethnic populations. Nevertheless, since some studies have shown that the sexual dimorphism of facial features is culturally stable $[61,62]$ and numerous studies have found the AQ to be culturally stable [63-65], we would anticipate similar AQ group differences in face morphology for other ethnic populations as well. An extension of the current study would be to investigate other sexually dimorphic physical characteristics such as voice $[66,67]$ and body composition $[68]$ in relation to autistic-like traits to determine whether the androgyny account applies to these physical features as well.

\section{Conclusions}

Bejerot et al. [26] provided preliminary evidence for the androgyny account using subjective ratings of faces of individuals with ASD and typically developed individuals. The findings of the current study extend the work of Bejerot et al. [26] by using objective measurements of facial features obtained from 3D images. Results of the current study provided further support for the androgyny account in which both males and females with high levels of autistic-like traits were found to typically present more androgynous (that is, less masculine for males and less feminine for females) facial features when compared to males and females with low levels of autistic-like traits.

\section{Abbreviations}

2D: two-dimensional; 3D: three-dimensional; AQ: Autism-spectrum Quotient; ASD: autism spectrum disorder; LDA: linear discriminant analysis.

\section{Competing interests}

The authors declare that they have no competing interests.

\section{Authors' contributions}

SZG was involved in facial analyses using computer algorithms and analysed the data. DWT collected and analysed the data and took the lead role in writing the manuscript. SRS was involved in data collection and analyses. All authors were involved in the editing of the manuscript. All authors read and approved the final manuscript.

\section{Acknowledgements}

Core Management of the Raine Study is funded by the University of Western Australia (UWA), Curtin University, the UWA Faculty of Medicine, Dentistry and Health Sciences, the Raine Medical Research Foundation, the Telethon Kids Institute, and the Women's and Infants Research Foundation. The authors acknowledge National Health and Medical Research Council (NHMRC) project grants (\#1021105, \#1021858, \#1044840) towards funding this data collection. The writing of this article was also supported by an NHMRC project grant (\#APP1003424) and the UWA Faculty of Engineering, Computing and Mathematics. SZG and DWT are supported by International Postgraduate Research Scholarships in Australia. SRS is partly supported by an Australian Research Council (ARC) Discovery grant (DP120104713). AM (DP 110102399) and FS (LP 110201008) are supported by ARC. AJOW is funded by a Career Development Fellowship from the NHMRC (\#1004065). PRE is supported by a NHMRC Senior Research Fellowship (\#513704). The authors are extremely grateful to the UWA participants, Raine Study participants and to the Raine Study team for cohort management and data collection.

\section{Author details}

'School of Computer Science and Software Engineering, University of Western Australia, 35 Stirling Highway, Crawley, 6009 Perth, WA, Australia. ${ }^{2}$ Neurocognitive Development Unit, School of Psychology, University of Western Australia, 35 Stirling Highway, Crawley, 6009 Perth, WA, Australia. ${ }^{3}$ School of Anatomy, Physiology and Human Biology, University of Western Australia, 35 Stirling Highway, Crawley, 6009 Perth, WA, Australia. ${ }^{4}$ School of Dentistry/Oral Health Centre of Western Australia, University of Western Australia, 35 Stirling Highway, Crawley, 6009 Perth, WA, Australia. ${ }^{5}$ Telethon Kids Institute, University of Western Australia, 100 Roberts Road, Subiaco, 6008 Perth, WA, Australia.

Received: 18 August 2014 Accepted: 2 April 2015

Published online: 15 April 2015

\section{References}

1. Allanson J, O'Hara R, Farkas L, Nair R. Anthropometric craniofacial pattern profiles in Down syndrome. Am J Med Genet. 1993;47:748-52. 
2. Hammond P, Hutton T, Allanson J, Buxton B, Campbell L, Clayton-Smith J, et al. Discriminating power of localized three-dimensional facial morphology. Am J Hum Genet. 2005;77:999-1010

3. American Psychiatric Association. Diagnostic and Statistical Manual of Mental Disorders. 5th ed. Arlington, VA: American Psychiatric Publishing; 2013.

4. Hammond P, Forster-Gibson C, Chudley A, Hutton T, Farrell S, McKenzie J, et al. Face-brain asymmetry in autism spectrum disorders. Mol Psychiatry. 2008;13:614-23.

5. Diewert V, Lozanoff S, Choy V. Computer reconstructions of human embryonic craniofacial morphology showing changes in relations between the face and brain during primary palate formation. J Craniofac Genet Dev Biol. 1993;13:193-201.

6. Marcucio R, Young N, Hu D, Hallgrimsson B. Mechanisms that underlie co-variation of the brain and face. Genesis. 2011:49:177-89.

7. Aldridge K, George I, Cole K, Austin J, Takahashi T, Duan Y, et al. Facia phenotypes in subgroups of prepubertal boys with autism spectrum disorders are correlated with clinical phenotypes. Molecular Autism. 2011;2:1-12.

8. Ozgen H, Hellemann G, Stellato R, Lahuis B, van Daalen E, Staal W, et al. Morphological features in children with autism spectrum disorders: a matched case-control study. J Autism Dev Disord. 2011;41:23-31.

9. Fombonne E. Epidemiology of pervasive developmental disorders. Pediatr Res. 2009;65:591-8.

10. Baron-Cohen S. The extreme male brain theory of autism. Trends Cogn Sci. 2002;6:248-54

11. Asperger H. Die, Autistisehen Psychopathen" im Kindesalter. Archiv für Psychiatrie und Nervenkrankheiten. 1944;117:76-136.

12. Frith U. Autism and Asperger syndrome. Cambridge: Cambridge University Press; 1991.

13. Auyeung B, Baron-Cohen S, Ashwin E, Knickmeyer R, Taylor K, Hackett G. Fetal testosterone and autistic traits. Br J Psychol. 2009;100:1-22.

14. Lutchmaya S, Baron-Cohen S, Raggatt P. Foetal testosterone and eye contact in 12-month-old human infants. Infant Behav Dev. 2002;25:327-35.

15. Chapman E, Baron-Cohen S, Auyeung B, Knickmeyer R, Taylor K, Hackett G. Fetal testosterone and empathy: evidence from the empathy quotient (EQ) and the "reading the mind in the eyes" test. Soc Neurosci. 2006;1:135-48.

16. Falter C, Plaisted K, Davis G. Visuo-spatial processing in autism-testing the predictions of extreme male brain theory. J Autism Dev Disord. 2008;38:507-15

17. Auyeung B, Knickmeyer R, Ashwin E, Taylor K, Hackett G, Baron-Cohen S Effects of fetal testosterone on visuospatial ability. Archives of Sex Behavior. 2012:41:571-81.

18. Lutchmaya S, Baron-Cohen S, Raggatt P, Knickmeyer R, Manning J. 2nd to 4th digit ratios, fetal testosterone and estradiol. Early Hum Dev. 2004;77:23-8.

19. Teatero $M$, Netley C. A critical review of the research on the extreme male brain theory and digit ratio (2D:4D). J Autism Dev Disord. 2013;43:2664-76.

20. Hönekopp J. Digit ratio 2D:4D in relation to autism spectrum disorders, empathising, and systemizing: a quantitative review. Autism Res. 2012:5:221-30.

21. Schmidtova E, Kelemenova S, Celec P, Ficek A, Ostatnikova D. Polymorphisms in genes involved in testosterone metabolism in Slovak autistic boys. Endocrinologist. 2010;20:245-9.

22. Ruta L, Ingudomnukul E, Taylor K, Chakrabarti B, Baron-Cohen S. Increased serum androstenedione in adults with autism spectrum conditions. Psychoneuroendocrinology. 2011;36:1154-63.

23. Schaefer K, Fink B, Mitteroecker P, Neave N, Bookstein F. Visualizing facial shape regression upon 2 nd to 4 th digit ratio and testosterone. Collegium Antropologicum. 2005;29:415-9.

24. Neave N, Laing S, Fink B, Manning J. Second to fourth digit ratio, testosterone and perceived male dominance. Proc R Soc B. 2003;270:2167-72.

25. Marečková K, Weinbrand Z, Chakravarty M, Lawrence C, Aleong R, Leonard $\mathrm{G}$, et al. Testosterone-mediated sex differences in the face shape during adolescence: subjective impressions and objective features. Horm Behav. 2011;60:681-90.

26. Bejerot S, Eriksson J, Bonde S, Carlström K, Humble M, Eriksson E. The extreme male brain revisited: gender coherence in adults with autism spectrum disorder. Br J Psychiatry. 2012;201:116-23.

27. Whitehouse A, Hickey M, Ronald A. Are autistic traits in the general population stable across development? PLoS One. 2011;6:1-8.
28. Constantino J, Todd R. Autistic traits in the general population: a twin study, Arch Gen Psychiatry. 2003;60:524-30.

29. Gilani S, Rooney K, Shafait F, Walters M, Mian A. Geometric facial gender scoring: objectivity of perception. PLoS One. 2014:9:1-12.

30. Farkas L, Eiben O, Sivkov S, Tompson B, Katic M, Forrest C. Anthropometric measurements of the facial framework in adulthood: age-related changes in eight age categories in 600 healthy white North Americans of European ancestry from 16 to 90 years of age. J Craniofac Surg. 2004;15:288-98.

31. Farkas $L$, Katic M, Forrest C. International anthropometric study of facial morphology in various ethnic groups/races. J Craniofac Surg. 2005;16:615-46.

32. Baron-Cohen S, Wheelwright S, Skinner R, Martin J, Clubley E. The autism spectrum quotient $(A Q)$ : evidence from Asperger syndrome/high functioning autism, males and females, scientists and mathematicians. J Autism Dev Disord. 2001;31:5-17.

33. Savran A, Sankur B, Bilge M. Comparative evaluation of 3D vs. $2 D$ modality for automatic detection of facial action units. Pattern Recogn. 2012;45:767-82.

34. Burton A, Bruce V, Dench N. What's the difference between men and women? Evidence from facial measurement. Perception. 1993;22:153-76.

35. Velemínská J, Bigoni L, Krajíček V, Borský J, Šmahelová D, Cagáňová V, et al. Surface facial modelling and allometry in relation to sexual dimorphism. J Comp Hum Biol. 2012;63:81-93.

36. Chakravarty M, Aleong R, Leonard G, Perron M, Pike G, Richer L, et al. Automated analysis of craniofacial morphology using magnetic resonance images. PLoS One. 2011;6:1-13.

37. McKnight CM, Newnham J, Stanley F, Mountain J, Landau L, Beilin L, et al. Birth of a cohort-the first 20 years of the Raine study. Med J Aust. 2012;197:608-10.

38. Aldridge K, Boyadjiev S, Capone G, DeLeon V, Richtsmeier J. Precision and error of three-dimensional phenotypic measures acquired from 3dMD photogrammetric images. Am J Med Genet. 2005:138:247-53.

39. Weinberg S, Scott N, Neiswanger K, Brandon C, Marazita M. Digital three-dimensional photogrammetry: evaluation of anthropometric precision and accuracy using a Genex 3D camera system. Cleft Palate Craniofac J. 2004:41:507-18.

40. Farkas L. Anthropometry of the head and face. 2nd ed. New York: Raven Press Ltd; 1994.

41. Duda R, Hart P, Stork D. Pattern classification and scene analysis. 2nd ed. Canada: Wiley; 2001

42. Russell-Smith S, Maybery M, Bayliss D. Are the autism and positive schizotypy spectra diametrically opposed in local versus global processing? J Autism Dev Disord. 2010;40:968-77.

43. Ruzich E, Allison C, Smith P, Watson P, Auyeung B, Ring H, et al. Measuring autistic traits in the general population: a systematic review of the Autism-spectrum Quotient (AQ) in a nonclinical population sample of 6,900 typical adult males and females. Molecular Autism. 2015;6:1-12

44. Whitehouse A, Mattes E, Maybery M, Dissanayake C, Sawyer M, Jones R, et al. Perinatal testosterone exposure and autistic-like traits in the general population: a longitudinal pregnancy-cohort study. J Neurodev Disord. 2012:4:1-12.

45. Russell-Smith S, Maybery M, Bayliss D. Relationships between autistic-like and schizotypy traits: an analysis using the autism spectrum quotient and oxford-Liverpool inventory of feelings and experiences. Personal Individ Differ. 2011:51:128-32.

46. Woodbury-Smith M, Robinson J, Wheelwright S, Baron-Cohen S. Screening adults for Asperger syndrome using the AQ: a preliminary study of its diagnostic validity in clinical practice. J Autism Dev Disord. 2005;35:331-5.

47. Grinter E, Maybery M, Van Beek P, Pellicano E, Badcock J, Badcock D. Global visual processing and self-rated autistic-like traits. J Autism Dev Disord. 2009:39:1278-90.

48. Jobe L, White $\mathrm{S}$. Loneliness, social relationships, and a broader autism phenotype in college students. Personal Individ Differ. 2007:42:1479-789.

49. Bruce V, Burton A, Hanna E, Healey P, Mason O, Coombes A, et al. Sex discrimination: how do we tell the difference between male and female faces? Perception. 1993;22:131-52.

50. Lai M, Lombardo M, Suckling J, Ruigrok A, Chakrabarti B, Ecker C, et al. Biological sex affects the neurobiology of autism. Brain. 2013;136:2799-815.

51. Scott $\mathrm{N}$, Jones $\mathrm{A}$, Kramer R, Ward R. Facial dimorphism in autistic quotient scores. Clin Psychol Sci. 2014;1:12 
52. Baron-Cohen S, Auyeung B, Nørgaard-Pedersen B, Hougaard D, Abdallah M, Melgaard $\mathrm{L}$, et al. Elevated fetal steriodogenic activity in autism. Mol Psychiatry. 2014;1:8.

53. Majewska M, Hill M, Urbanowicz E, Rok-Bujko P, Bieńkowski P, Namyslowska I, et al. Marked elevation of adrenal steroids, especially androgens, in saliva of prepubertal autistic children. Eur Child Adolesc Psychiatry. 2014;23:485-98.

54. Takagishi H, Takahashi T, Yamagishi T, Shinada M, Inukai K, Tanida S, et al. Salivary testosterone levels and autism-spectrum quotient in adults. Neuroendocrinol Lett. 2010;31:837-41.

55. Petersen A. Physical androgyny and cognitive functioning in adolescence. Dev Psychol. 1976;12:524-33.

56. Poswillo D. The aetiology and pathogenesis of craniofacial deformity Development. 1988;103:207-12.

57. Anderson G, Galfin T, Xu W, Aoto J, Malenka R, Südhof T. Candidate autism gene screen identifies critical role for cell-adhesion molecule CASPR2 in dendritic arborization and spine development. Proc Natl Acad Sci U S A. 2012:44:18120-5.

58. Lord C, Rutter M, Dilavore P, Risi S, Gotham K, Bishop S. Autism diagnostic observation schedule. 2nd ed. TorranceWestern: Psychological Services; 2012.

59. Lord C, Rutter M, Couteur A. Autism diagnostic interview-revised: a revised version of a diagnostic interview for caregivers of individuals with possible pervasive developmental disorders. J Autism Dev Disord. 1994;24:659-85.

60. Robinson E, Koenen K, McCormick M, Munir K, Hallett V, Happé F, et al. Evidence that autistic traits show the same etiology in the general population and at the quantitative extremes (5\%,25\%, and 1\%). Arch Gen Psychiatry. 2011;68:1113-21.

61. Stephen I, Scott I, Coetzee V, Pound N, Perrett D, Penton-Voak I. Crosscultural effects of color, but not morphological masculinity, on perceived attractiveness of men's faces. Evol Hum Behav. 2012;33:260-7.

62. Gilani S, Shafait F, Mian A. Hobart biologically significant facial landmarks: how significant are they for gender classification? In Digital Image Computing: Techniques and Application (DICTA). Edited by IEEE. 2013;2013:1-8. International conference: 26-28 November 2013.

63. Sonié S, Kassai B, Pirat E, Bain P, Robinson J, Gomot M, et al. The french version of the autism-spectrum quotient in adolescents: a cross-cultural validation study. J Autism Dev Disord. 2013;43:1178-83.

64. Ruta L, Mazzone D, Mazzone L, Wheelwright S, Baron-Cohen S. The Autismspectrum Quotient-Italian version: a cross-cultural confirmation of the broader autism phenotype. J Autism Dev Disord. 2012;42:625-33.

65. Wakabayashi A, Baron-Cohen S, Uchiyama T, Yoshida Y, Kuroda M, Wheelwright S. Empathizing and systemizing in adults with and without autism spectrum conditions: cross-cultural stability. J Autism Dev Disord. 2007;37:1823-32.

66. Lieberman P. Some aspects of dimorphism and human speech. Hum Evol. 1986;1:67-75.

67. Voperian H, Wang S, Schimek E, Durtschi R, Kent R, Gentry L, et al. Developmental sexual dimorphism of the oral and pharyngeal portions of the vocal tract: an imaging study. J Speech Lang Hear Res. 2011:54:995-1010.

68. Wells J. Sexual dimorphism of body composition. Best Practice Res Clin Endocrinol Metab. 2007;21:415-30.

69. Burns R, Burns R. Discriminant analysis. In business research methods and statistics using SPSS. London: SAGE Publication Ltd; 2009.

\section{Submit your next manuscript to BioMed Central and take full advantage of:}

- Convenient online submission

- Thorough peer review

- No space constraints or color figure charges

- Immediate publication on acceptance

- Inclusion in PubMed, CAS, Scopus and Google Scholar

- Research which is freely available for redistribution

Submit your manuscript at www.biomedcentral.com/submit 